\title{
PERIODIC POINTS OF CONTINUOUS MAPS AND LINDEMANN'S INDEPENDENCE THEOREM FOR EXPONENTIALS
}

DEAN A. NEUMANN

\begin{abstract}
We give a simple proof of the sufficiency of known conditions for the existence of periodic points of a continuous map, using a classical theorem of Lindemann on transcendental numbers.
\end{abstract}

The purpose of this note is to give a very simple proof of a generalization of the following well-known theorem of F. B. Fuller:

THEOREM 1 (FULLER). If $\phi$ is a self-homeomorphism of a finite complex $X$, and $\chi(X) \neq 0$, then $\phi$ has a periodic point.

We consider a space $X$ for which the Lefschetz Fixed Point Theorem is valid, and a map $\phi: X \rightarrow X$. Let $\phi_{* q}: H_{q}(X) \rightarrow H_{q}(X)(q \geqslant 0)$ denote the induced maps of the rational homology of $X$; set $\lambda_{0}=0$, and let $\lambda_{1}, \ldots, \lambda_{n}$ be the distinct nonzero eigenvalues of the $\phi_{* q}$; let $m_{q}\left(\lambda_{j}\right)$ be the multiplicity of $\lambda_{j}$ in $\phi_{* q}(j=0, \ldots, n)$. We then have:

THEOREM 2. If $\phi$ is a self-map of a Lefschetz space $X$ and $\phi$ has no periodic points, then

$$
\sum_{q}(-1)^{q} m_{q}\left(\lambda_{0}\right)=\chi(X) \text { and } \sum_{q}(-1)^{q} m_{q}\left(\lambda_{j}\right)=0 \quad(j=1, \ldots, n) .
$$

Theorem 1 has been considerably extended by Fuller [2] and others: the survey [1] of Fadell contains an elegant exposition of these results. Theorem 2 also follows from these extensions, but our direct proof is somewhat simpler.

Proof of Theorem 2. Let $m$ be the maximum dimension for which $H_{q}(X) \neq 0$. For $q=0, \ldots, m$, let $A_{q}$ be a matrix representing $\phi_{* q}$ with respect to some fixed basis for $H_{q}(X)$. Define

$$
B_{k}=\operatorname{diag}\left[A_{0}^{k},-A_{1}^{k}, \ldots,(-1)^{m} A_{m}^{k}\right]
$$

for $k \geqslant 0$, and set

$$
E=\sum_{k=0}^{\infty} \frac{1}{k !} B_{k}
$$

Received by the editors May 24, 1976.

AMS (MOS) subject classifications (1970). Primary 55C20; Secondary 54H25.

Key words and phrases. Lefschetz number, fixed points for iterates.

Copyright $\odot$ 1977, American Mathematical Society 
Note that $\operatorname{Tr}\left(B_{0}\right)=\chi(X)$, and, for $k \geqslant 1, \operatorname{Tr}\left(B_{k}\right)=\Lambda\left(\phi^{k}\right)$, the Lefschetz number of $\phi^{k}$. If $\phi$ has no periodic points then, by the Lefschetz Fixed Point Theorem, $\operatorname{Tr}\left(B_{k}\right)=0$ for $k \geqslant 1$. We then have, equating traces in (1):

$$
\sum_{j=0}^{n} \sum_{q=0}^{m}(-1)^{q} m_{q}\left(\lambda_{j}\right) e^{\lambda_{j}}=\chi(X) \cdot e^{\lambda_{0}} .
$$

But the $A_{q}$ are rational matrices, so the eigenvalues $\lambda_{0}, \ldots, \lambda_{n}$ are distinct algebraic numbers, and we can apply the theorem of Lindemann ([3, p. 117], e.g.) that asserts the linear independence over the field of algebraic numbers of $e^{\lambda_{0}}, e^{\lambda_{1}}, \ldots, e^{\lambda_{n}}$, to conclude that each of the coefficients in equation (2) vanishes. This is just the conclusion of Theorem 2 .

\section{BIBLIOGRAPHY}

1. E. R. Fadell, Recent results in the fixed point theory of continuous maps, Bull. Amer. Math. Soc. 76 (1970), 10-29. MR 42 \#6816.

2. F. B. Fuller, Periodic trajectories of a one-parameter semigroup, Bull. Amer. Math. Soc. 69 (1963), 409-410. MR 26 \#4002.

3. Ivan Niven, Irrational numbers, Math. Assoc. Amer. Carus Monograph No. 11, Wiley, New York, 1956. MR 18, 195.

Department of Mathematics, Bowling Green State University, Bowling Green, Ohio 43403 